\title{
Transplantes e Povos Indígenas: uma Abordagem Inicial
}

\author{
Estevão Rafael Fernandes ${ }^{1}$ (D), Ana Karoline Nóbrega Cavalcanti ${ }^{2}$
} 1. Universidade Federal de Rondônia RóR -
Porto Velho (RO), Brasil.

2. Secretaria de Estado de Saúde - Porto Velho (RO), Brasil.

doi https://doi.org/10.53855/bjt.v25i1.439_pt

Autor correspondente:

estevaofernandes@gmail.com

Editor de Seção

Ilka Boin

Recebido

Jan. 12, 2022

Aprovado

Jan. 13, 2022

Conflito de interesse

Nada a declarar

Como Citar

Fernandes ER, Cavalcanti AKN. Transplantes e povos indígenas: uma abordagem inicial. BJT. 2022; 25(01): e0122. https://doi.org/10. 53855/bjt.v25i1.439_pt

eISSN

2764-1589

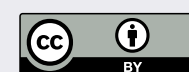

Resumo: Este artigo indica um conjunto de questões relativas aos transplantes envolvendo pessoas indígenas. Trata-se de um tema não abordado na literatura do país e para o qual é relevante lançar um olhar mais detido, dados os aspectos não apenas médicos, mas também bioéticos, sociocosmológicos, religiosos, sociais e culturais. Desde uma perspectiva interdisciplinar, buscou-se aqui chamar a atenção para a importância de se levar em conta aspectos relativos à constituição da pessoa indígena; implicações referentes ao consentimento informado; e a necessidade de um olhar sensível para as diferenças interculturais implicadas. A conclusão foi que se faz necessária a formação de um protocolo em conjunto com organizações indígenas e órgãos oficiais que considere as especificidades culturais. Além disso, um levantamento tanto das iniciativas institucionais em outros países quanto da literatura sobre a temática ainda é tarefa a ser feita no Brasil.

Descritores: Povos Indígenas; Saúde de Populações Indígenas; Obtenção de Tecidos e Órgãos.

\section{INTRODUÇÃO}

O Brasil é um país diverso etnicamente. Em seu território, encontram-se mais de 300 etnias, falando cerca de 280 línguas diferentes. Apesar de esses indígenas povoarem o imaginário como intocados, em meio à Amazônia, aproximadamente $40 \%$ da população indígena brasileira habita em ambientes urbanos, segundo estimativas do Instituto Brasileiro de Geografia e Estatística. ${ }^{1}$

Do ponto de vista sanitário, vários desses povos já enfrentam a chamada "transição epidemiológica", 2 com um número crescente de mortes causadas por doenças crônicas - como obesidade, diabetes, problemas renais, problemas do coração, por exemplo -, consequência direta ou indireta do contato com a sociedade envolvente. Da mesma forma, problemas como falta de saneamento, poluição advinda de garimpos ou contato direto com agrotóxicos ${ }^{3}$ atingem cotidiana e sistematicamente comunidades indígenas, incluindo povos isolados. Nesse contexto, pode-se afirmar ser questão de tempo para haver uma demanda crescente por transplantes por parte dessa população, algo até aqui pouco enfrentado tanto pela literatura do país quanto por gestores e legisladores.

Não se trata mais de considerar os povos indígenas em território nacional como alheios às demandas e aos problemas mais gerais da população como um todo, mas, ao contrário, de buscar estender a eles toda a assistência à qual têm direito, desde que respeitados aspectos específicos de suas culturas. 


\section{DISCUSSÃO}

Essas considerações iniciais levam a uma pergunta: que questões devem ser levadas em conta nos transplantes envolvendo povos indígenas?

Dados os limites deste texto, pensamos ser importante salientar sobretudo três aspectos haja vista esse problema. Em primeiro lugar, tem-se as especificidades ao se trabalhar em outra perspectiva de pessoa. Dito isso brevemente - esse ponto será desenvolvido adiante -, no Ocidente a noção de pessoa se confunde com a de Homo sapiens. Nos povos indígenas, por outro lado, como as formas de classificação do mundo são diversas e não se fundamentam nos nossos pressupostos biologizantes, um sujeito torna-se pessoa por intermédio de uma série de complexas relações sociais e rituais. O corpo não é, nessas sociedades, mero artefato físico, mas a soma de relações sociais e cosmológicas compreendidas tão somente com base em suas culturas.

Em segundo lugar, faz-se necessário indicar implicações práticas quanto ao consentimento informado: como obtê-lo da melhor forma possível, em um contexto intercultural?

Finalmente, buscou-se chamar a atenção para a importância de um olhar sensível para essas diferenças culturais e para um pressuposto significativo tratando-se desse tipo de problemática: a intervenção em um corpo indígena repercute em toda a sua comunidade de várias maneiras diferentes, e, no que tange a transplantes, essa intervenção se complexifica consideravelmente. Concluiu-se aqui ser relevante a constituição, em conjunto com organizações indígenas e órgãos indigenistas, de um protocolo que regulamente transplantes em contextos interculturais, como modo de resguardar tanto comunidades indígenas quanto equipes médicas por todo o país.

Feitas essas considerações, é interessante indicar como a problemática transplantes envolvendo pessoas indígenas não causa alerta entre pesquisadores no Brasil. Buscando-se nas principais bases de dados da área da saúde (PubMed, Literatura Latino-Americana e do Caribe em Ciências da Saúde - Lilacs, Biblioteca Eletrônica Científica Online - SciELO, entre outras), por meio de descritores tais como indígenas ou povos indígenas e transplante, o que se viu foi a completa falta de textos produzidos sobre o tema - mesmo em referências importantes acerca de saúde indígena no país. ${ }^{4}$ Além disso, ainda que disponhamos de uma política relativamente avançada direcionando o Subsistema de Atenção à Saúde Indígena, é importante indicar que a Política Nacional de Atenção à Saúde dos Povos Indígenas, aprovada em 2002, não faz menção à palavra transplante nenhuma vez sequer em todo o seu texto.

Esse vazio a respeito do tema no contexto brasileiro chama a atenção em relação ao que se produz fora do país, na academia ou além dela. Uma rápida busca usando-se os mesmos descritores leva a vários textos sobre o tema em diferentes contextos nacionais e culturais - alguns exemplos deixam essa diversidade clara. ${ }^{5-11}$

Já institucionalmente, há várias iniciativas oficiais bem-sucedidas sendo desenvolvidas ou em curso em outros países no sentido de se pensar transplantes em povos indígenas. Basta dizer que o Office of Minority Health do U.S. Department of Health and Human Services - o equivalente ao nosso Ministério da Saúde - possui um website específico sobre doação de órgãos para nativos americanos dos Estados Unidos continental e do Alasca. ${ }^{12}$ Naquele país, conforme informações disponibilizadas no site, foram realizados 280 transplantes envolvendo pessoas indígenas em 2020, quando havia mais de 900 americanos nativos aguardando transplantes de rins, fígado, pulmão e coração.

No Canadá, por sua vez, há aplicativos de celular voltados para as pessoas pertencentes a first nations, como o Kidney Check Program, ou iniciativas para discutir como o sistema de saúde pode acolher pacientes indígenas e suas famílias nas ações referentes a transplantes de órgãos - como no encontro virtual ocorrido em dezembro de 2021, promovido em colaboração da Network Environments for Indigenous Health Research National Coordinating Centre, do First Nations and Métis Organ Donation and Transplantation Network e da CanSOLVE CKD Network (Fig. 1).

Não se dispõe aqui de espaço para analisar, de forma detida e merecida, as diferenças entre as políticas indigenistas nesses contextos nacionais - espera-se fazê-lo futuramente, bem como um exercício de revisão dessa literatura. Basta, aqui, apontar de modo preliminar que tal sensibilidade é fruto não apenas de um longo percurso de demandas dos movimentos indígenas organizados nesses países, mas também de uma relação diferente em termos institucionais com tais povos por parte desses Estados e construída historicamente. ${ }^{14}$

Contudo, há um ponto específico que interessa diretamente a quem busca pensar transplantes e povos indígenas desde uma perspectiva legal. No Brasil, temos uma legislação indigenista nascida em pleno regime militar - o Estatuto do Índio é de 1973 - baseada na ideia de tutela. O objetivo era "integrar os índios pacificamente à União Nacional", exercendo sobre essas comunidades todo o controle possível, sobretudo à luz da Doutrina de Segurança Nacional. ${ }^{15} \mathrm{O}$ conceito de uma autonomia cultural apenas veio a ser respeitado com a Constituição Federal de 1988, mas, ainda assim, o respeito às culturas indígenas, previsto no Artigo 231 da Carta Constitucional, fica submetido, até os dias de hoje, ao crivo do órgão tutor - ou seja, a Fundação Nacional do Índio (Funai). Em suma, isso significa que qualquer intervenção, como um transplante, por exemplo, dada a falta de uma previsão legal específica ou de protocolos constituídos de forma clara e colaborativa, pode transformar-se facilmente em um campo de batalha burocrático e jurídico envolvendo Funai, Advocacia-Geral da União, Ministérios da Saúde, dos Direitos Humanos, da Justiça, Público, organizações indígenas, entre outros. A ideia de um protocolo viria não apenas a respaldar as equipes de saúde, mas também de resguardar a cultura desses povos e suas próprias noções de saúde, doença e pessoa. 


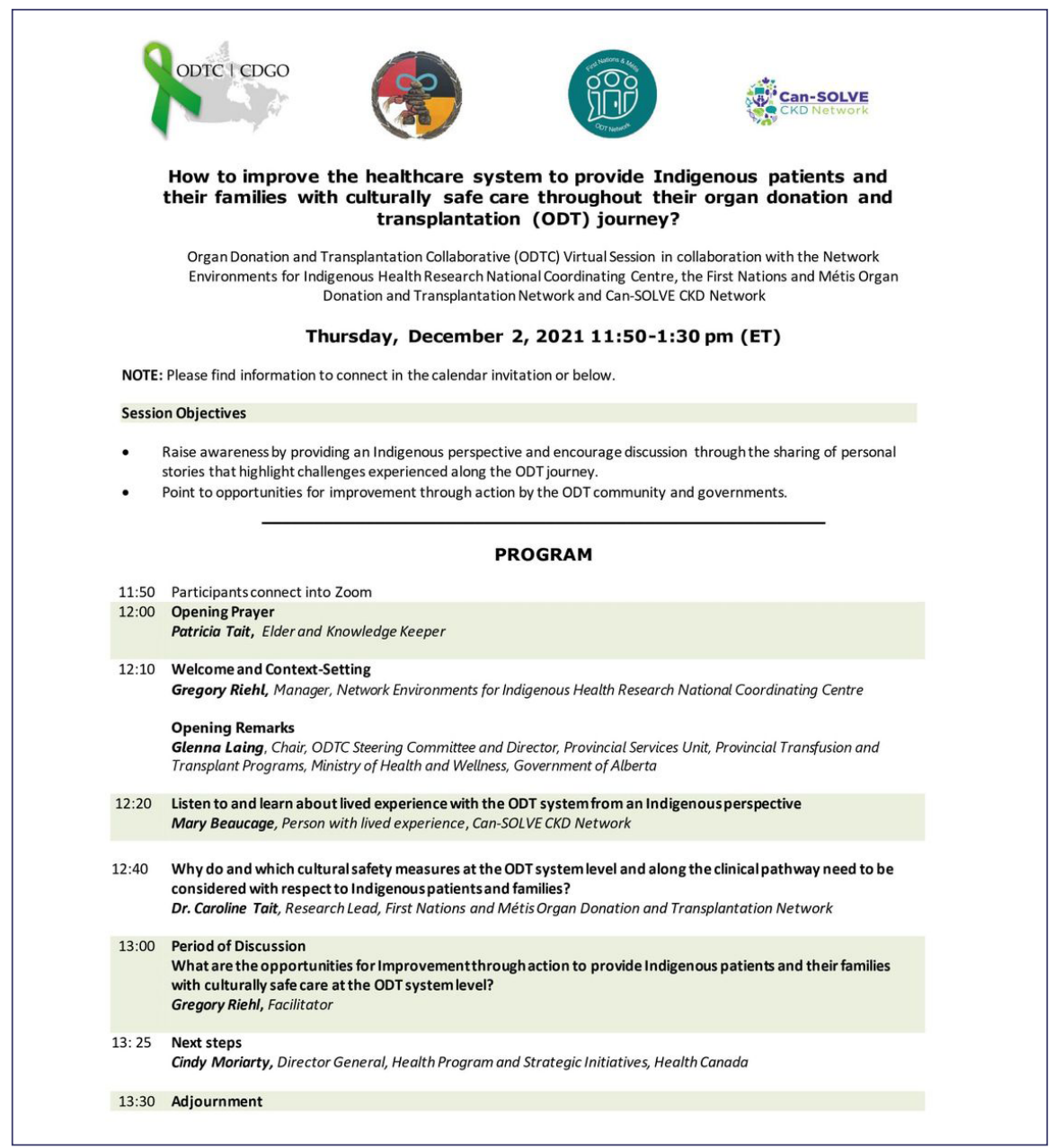

Fonte: First Nations and Métis Organ Donation and Transplantation Network. ${ }^{13}$

Figura 1. Organ donation and transplantation collaborative, Canadá.

Não se trata, é importante salientar, tão somente de respeitar as culturas indígenas - ou seja, um pressuposto moral, e sim também de uma norma estabelecida desde a assinatura da Convenção 169 da Organização Internacional do Trabalho (OIT), transformada em lei no Brasil em 2004, firmando a obrigatoriedade da consulta prévia e informada aos povos indígenas sobre decisões que impactem diretamente suas vidas.

O ponto aqui é: de que maneira um transplante, por exemplo, impactaria essas sociedades haja vista a perspectiva de suas culturas e como mitigar esses impactos?

Como foi dito anteriormente, a noção de pessoa entre os povos indígenas é um bom ponto de partida para se compreender esse tipo de problema. A questão é investigada no âmbito da antropologia brasileira há mais de 40 anos ${ }^{16}$ e pode ser resumida da seguinte forma: o corpo ameríndio é construído de um conjunto de relações simbólicas, rituais, de parentesco, de nominação. Ou seja, o sujeito torna-se um lugar de encontro de diversas relações sociais, sendo construído e compreendido pelas categorias de pensamento nativas. $\mathrm{O}$ que os ocidentais entendem como indivíduo nos povos indígenas dá lugar a uma unidade social que opera em relação às redes de parentesco, mitologias, cosmologias e perspectivas nativas de natureza, por exemplo. Uma vez que o sujeito é esse cruzamento de cadeias simbólicas, ele deve ser compreendido com base nelas, de tal modo que uma ação sobre o corpo nativo é, via de regra, algo que atinge todo o corpus daquela sociedade.

Como isso funciona na prática? Um dos autores deste texto teve a oportunidade de analisar, há alguns anos, ${ }^{17}$ ideologias em torno da concepção de pessoa entre os índios jê do Brasil Central, permitindo dar um exemplo de como operam essas concepções nesses povos. Em resumo, em algumas sociedades, o feto é visto como substância ligada diretamente ao pai e aos seus parentes consanguíneos, de tal forma que qualquer alimento ingerido por seu pai, tios, avô paterno e primos paralelos repercute no corpo da criança por vários anos após o nascimento. Isso faz com que haja uma série de restrições e tabus alimentares por parte dessas pessoas, a fim de resguardar sua saúde: se um tio se alimenta de carne de tatu, a criança comerá terra; caso seu pai coma feijão, sua pele ficará preta etc. É frequente, por exemplo, buscar-se um culpado por quebrar esses tabus em caso de doenças mais graves, 
de modo a restabelecer sua saúde. Crianças nessas sociedades passam anos sem um nome próprio, pois precisam esperar seus corpos terem força suficiente para aguentar um nome - ou seja, terem relativa autonomia como sujeitos -, e ainda assim há várias mudanças de nome ao longo da vida, marcando modificações importantes no status dessas pessoas: novos marcos em seus corpos, nos rituais dos quais participam, dos papéis que ocupam em suas comunidades, entre outros.

Outro exemplo também bastante conhecido e bem documentado diz respeito às amostras de sangue dos indígenas ianomâmi (Roraima/Amazonas) levadas por pesquisadores dos Estados Unidos nos anos de 1960 e 70. Por mais de quatro décadas, as lideranças ianomâmi buscaram recuperar essas amostras a fim de aplacarem os espíritos daquelas pessoas já falecidas cujo sangue fora retirado. Naquela cultura, os mortos devem ser cremados em uma série de complexos rituais, e o sangue congelado em alguma universidade impedia tal cerimônia, uma vez que parte daquelas pessoas não havia passado por esses ritos.

Exemplos nesse sentido são legião e indicam o que se tem tentado apontar aqui: processos de saúde, doença, corporalidade, cura e cuidados entre povos indígenas, de modo geral, são parte relevante da vida coletiva dessas sociedades e apenas podem ser compreendidos à luz de suas culturas. Por ser a pessoa indígena uma constituição social, essas intervenções possuem também repercussão social.

Isso leva a outro ponto: as repercussões bioéticas dessas questões na prática. Muito se conhece sobre a Resolução do Conselho Nacional de Saúde (CNS) no 466/2012, a qual estabelece as diretrizes para pesquisas em seres humanos, mas chama-se a atenção aqui para outras duas normas, relativamente pouco conhecidas. A primeira, $\mathrm{n}^{\circ} 304 / 2000$, ainda em vigor (mesmo tendo sido feita sob vigência da Resolução no 196/1996), diz respeito à ética em pesquisa especificamente com povos indígenas. A segunda norma é a Resolução CNS no 510/2016, que normatiza as ciências humanas nas pesquisas que possam acarretar riscos à vida cotidiana.

Essas duas normas são trazidas aqui por darem algumas diretrizes importantes no tocante a abordagens possíveis na busca por consentimento, em caso de transplante envolvendo pessoas indígenas. Tais diretrizes são sugestões, podendo ser readequadas, sendo o ideal - repete-se - a criação de um protocolo específico voltado para essas populações. Questões importantes tais como o respeito às particularidades culturais e às esferas de decisão nativas se encontram bem estabelecidas, mas falta, por exemplo, a previsão no uso de intérpretes ou tradutores para auxiliar naqueles procedimentos cujos envolvidos não possuam conhecimento pleno da língua portuguesa. Por mais que pareça preciosismo, a título de comparação, algumas delegacias de atendimento à mulher do país possuem mulheres intérpretes a fim de auxiliar no acolhimento das indígenas vítimas de violência. Afinal, mesmo aquelas que moram próximas a núcleos urbanos não necessariamente conseguem comunicar-se de maneira integral em português.

\section{CONCLUSÃO}

Viu-se que o transplante envolvendo povos indígenas no Brasil é ainda algo a ser enfrentado tanto por legisladores quanto por gestores e pesquisadores. Ainda que haja um acúmulo de literatura e algumas iniciativas em outros países nesse sentido, o tema não tem suscitado maiores reflexões em terras brasileiras. Este artigo é um primeiro passo nessa direção. Uma de nossas hipóteses é a de que a transição epidemiológica registrada em várias dessas comunidades e problemas sociais e ambientais diversos farão surgir, brevemente, demanda por transplantes por parte desses povos, a exemplo do que ocorre em outros países.

Ao longo deste texto, buscou-se mostrar, ainda que resumidamente, como questões culturais possuem implicações diretas ao se pensar transplante em uma perspectiva intercultural: corporalidade e pessoa ameríndias são parte de um conjunto complexo de relações sociais e culturais as quais devem ser respeitadas e consideradas nesse tipo de intervenção. Entretanto, dada essa complexidade, sugere-se aqui a construção, em parceria com organizações indígenas e órgãos de Estado, de um protocolo de transplantes que leve em conta essas especificidades.

Além disso, parece urgente a tarefa de realizar-se um levantamento detalhado da literatura sobre transplantes em povos indígenas em outros contextos nacionais, bem como das iniciativas executadas em outros países, de modo a aprender com essas experiências.

\section{CONTRIBUIÇÃO DOS AUTORES}

Contribuições científicas e intelectuais substantivas para o estudo: Fernandes ER and Cavalcanti AKN; Concepção: Fernandes ER and Cavalcanti AKN.

\section{DISPONIBILIDADE DE DADOS DE PESQUISA}

Todos os dados foram gerados ou analisados no presente estudo. 


\section{FINANCIAMENTO}

Não se aplica.

\section{AGRADECIMENTOS}

Não se aplica.

\section{REFERÊNCIAS}

1. Instituto Brasileiro de Geografia e Estatística (IBGE). Indígenas. Gráficos e tabelas [Internet]. Brasil: IBGE [acessado em 17 jan. 2022]. Disponível em: https://indigenas.ibge.gov.br/graficos-e-tabelas-2.html

2. Coimbra Jr. CE, Flowers NM, Salzano F, Santos RV. Xavante in transition: health, ecology and bioanthropology in Central Brazil. Ann Arbor: University of Michigan Press; 2002.

3. Gonçalves GMS, Gurgel IGD, Costa AM, Almeida LR, Lima TFP, Silva E. Uso de agrotóxicos e a relação com a saúde na etnia Xukuru do Ororubá, Pernambuco, Brasil. Saúde Soc. 2012;21(4):1001-12. https://doi.org/10.1590/S0104-12902012000400017

4. Buchillet D. Bibliografia crítica da saúde indígena no Brasil (1844-2006). Quito: Abya-Yala; 2007.

5. Howson P, Irish AB, D’orsogna L, Chakera A, Swaminathan R, Perry G, et al. Allograft and Patient Outcomes Between Indigenous and Nonindigenous Kidney Transplant Recipients. Transplantation. 2020;104(4):847-55. https://doi.org/10.1097/ TP.0000000000002891

6. Barraclough KA, Grace BS, Lawton PMSP, McDonald SP. Residential location and kidney transplant outcomes in indigenous compared with nonindigenous Australians. Transplantation. 2016;100(10):2168-76. https://doi.org/10.1097/ TP.0000000000001007

7. Keddis MT, Sharma A, Ilyas M, Zhang N, Khamash H, Leischow SJ, et al. Transplant center assessment of the inequity in the kidney transplant process and outcomes for the Indigenous American patients. PLoS One. 2018;13(11):e0207819. https://doi. org/10.1371/journal.pone.0207819

8. Khanal N, Lawton PD, Cass A, McDonald SP. Disparity of access to kidney transplantation by Indigenous and non-Indigenous Australians. Med J Aust. 2018;209(6):261-6. https://doi.org/10.5694/mja18.00304

9. Walker RC, Abel S, Reynolds A, Palmer SC, Walker C, Tipene-Leach DC. Experiences, perspectives and values of Indigenous peoples regarding kidney transplantation: systematic review and thematic synthesis of qualitative studies. Int J Equity Health. 2019;18:204. https://doi.org/10.1186/s12939-019-1115-y

10. Majoni SW, Ullah S, Collett J, Hughes JT, McDonald S. Weight change trajectories in Aboriginal and Torres Strait islander Australians after kidney transplantation: a cohort analysis using the Australia and New Zealand Dialysis and Transplant registry (ANZDATA). BMC Nephrol. 2019;20:232. https://doi.org/10.1186/s12882-019-1411-1

11. Boan P, Swaminathan R, Irish A. Infectious complications in indigenous renal transplant recipients in Western Australia. Intern Med J. 2017;47(6):648-55. https://doi.org/10.1111/imj.13450

12. U.S. Departament of Health and Human Services. Organ Donation and American Indians/Alaska Natives. Estados Unidos: U.S. Departament of Health and Human Services, Office of Minority Health; 2021.

13. First Nations and Métis Organ Donation and Transplantation Network. Facebook [Internet]. [acessado em jan. 2022]. Disponível em: https://www.facebook.com/pg/FirstNationsMetisODT

14. Fernandes ER. Descolonizando sexualidades: enquadramentos coloniais e homossexualidade indígena no Brasil e nos Estados Unidos. Brasília: Editora Universidade de Brasília; 2020. https://doi.org/10.26512/9786558460442

15. Lima ACS. Um grande cerco de paz: poder tutelar, indianidade e formação do Estado no Brasil. Petrópolis: Vozes; 1995.

16. Seeger A, Matta R, Viveiros de Castro E. A construção da pessoa nas sociedades indígenas brasileiras. Boletim do Museu Nacional. 1979:2-19. Série Antropologia.

17. Fernandes ER. Do Tsihuri ao Waradzu: o que as ideologias xavante de concepção, substância e formação da pessoa nos dizem sobre o estatuto ontológico do outro? Horiz Antropol. 2010;16(34):453-77. https://doi.org/10.1590/S0104-71832010000200019 